\title{
Furosemide and Albumin for Diuresis of Edema (FADE): a parallel-group, blinded, randomized controlled pilot trial
}

Simon JW Oczkowski MD MHSc ${ }^{1,2}$, Lisa Klotz BSc ${ }^{2}$, lan Mazzetti MD ${ }^{3}$, Fayez Alshamsi MD ${ }^{4}$, Mei Lin Chen BHSc ${ }^{5}$,

Gary Foster $\mathrm{PhD}^{6,7}$, Maureen Meade MD MSc ${ }^{1,2,6}$, Cindy Hamielec $\mathrm{MD}^{1,2}$

1.Department of Medicine, McMaster University 2.Hamilton Health Sciences 3.Schulich School of Medicine and Dentistry, Western University 4.United Arab Emirates University

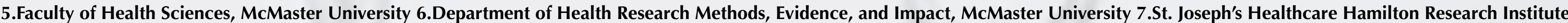

\section{Background}

Fluid overload is associated with morbidity and mortality in critically ill patients. Adding hyperoncotic albumin to standard treatment with diuretics may improve diuresis and facilitate liberation from mechanical ventilation.

\section{Objectives}

We conducted a pilot study to assess the feasibility of a definitive randomized controlled trial testing the effectiveness of hyperoncotic albumin as an adjunct to diuretics in critically ill adults.

\section{Materials \& Methods}

At two medical/neurotrauma ICUs in Canada, we randomized hemodynamically stable, hypoalbuminemic adults, judged to require diuresis, to receive either $25 \%$ albumin or $0.9 \%$ saline placebo, 100 $\mathrm{mL}$ twice daily for up to six doses, within 2 hours of prescribed furosemide. Patients, bedside staff, and research staff were blinded.

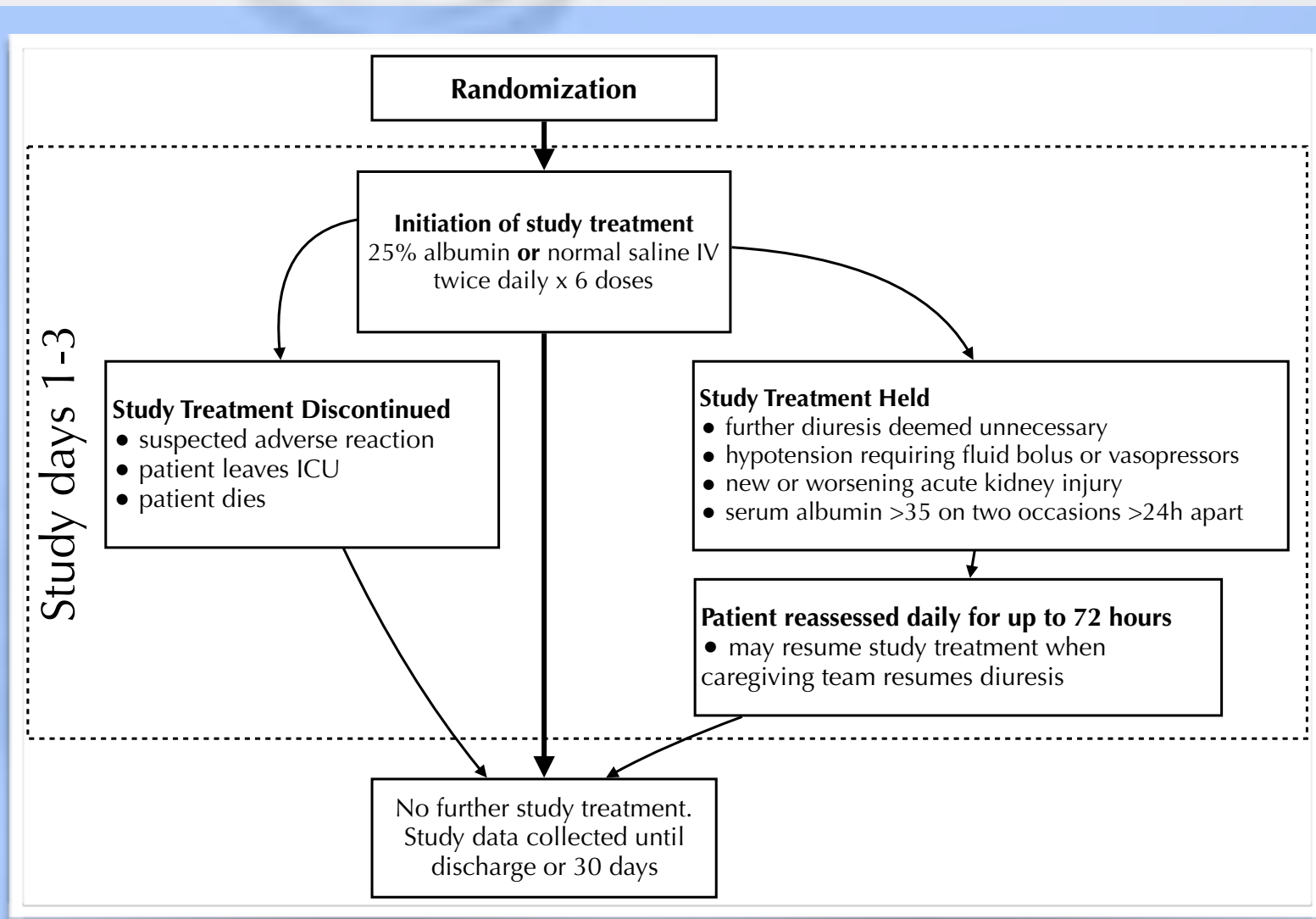

We specified five criteria for study feasibility: enrolment of $50 \%$ of eligible patients; enrolment of >I patient/week; administration of the first dose of study treatment within 2 hours of diuretics in $85 \%$ of patients; completion of study regimen in $80 \%$ of patients; and avoidance of open label $25 \%$ albumin in $85 \%$ of patients.

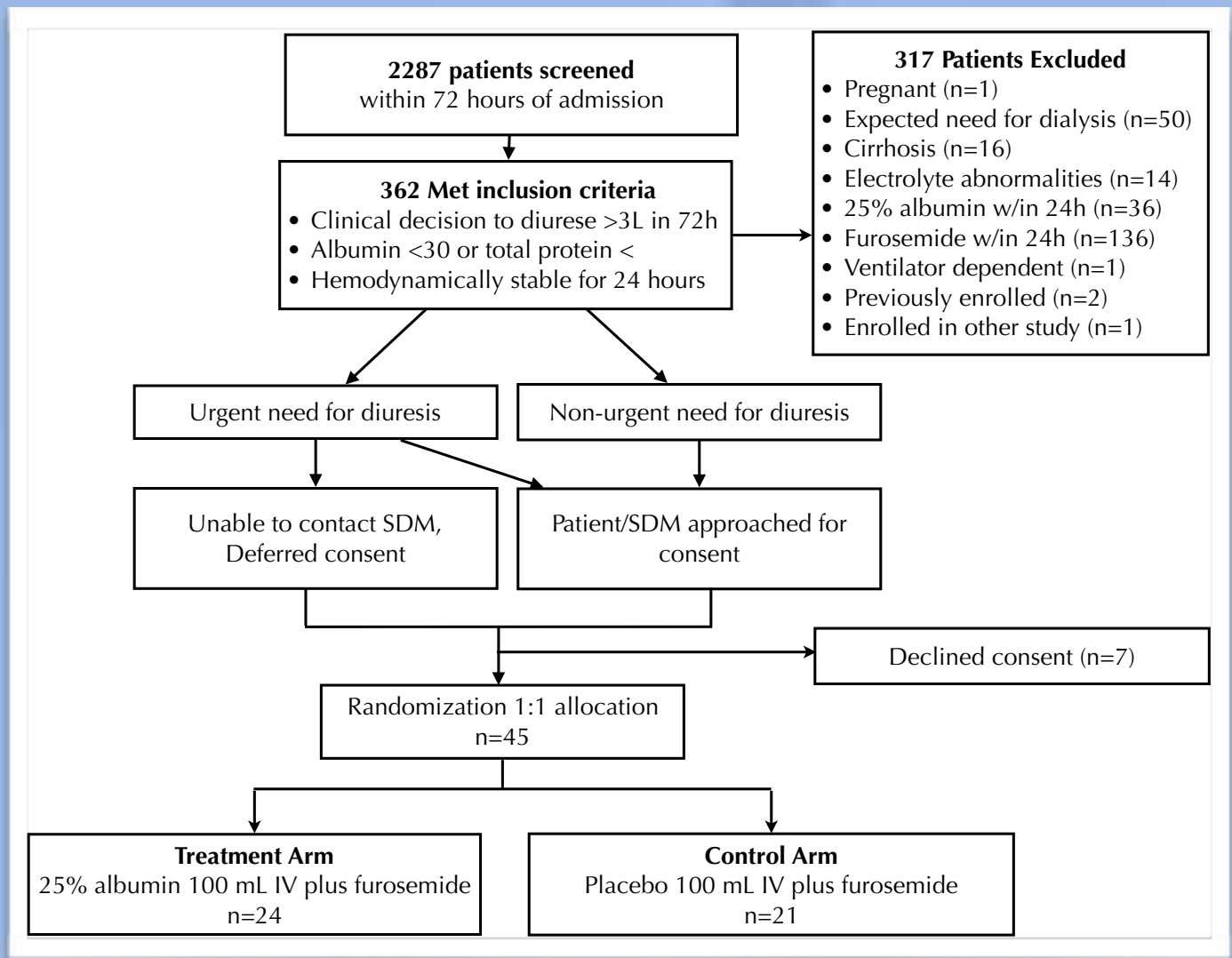

We successfully collected all clinical outcome data for the albumin and placebo groups, including:

- ventilator free days (median [QI, Q3]): 4.0 days $[0.0,23.5]$ v. 13.0 days $[0.0,21.0])$

- length of ICU stay (median [QI, Q3]): I8.25 days $[$ II.3, 38.0] v. 16.6 days $[11.2,26.2])$

- 30-day mortality $(6 / 24$ v. $2 / 21)$

No significant harms were identified by the clinical or research teams. Interruption of study treatment occurred in 8/24 patients receiving albumin, compared to $4 / 2$ I patients receiving placebo.

\section{Results}

From September 2014 to October 2016 we randomized $85 \%$ of eligible patients (24 albumin, 21 placebo). Due to slow recruitment ( 0.42 patients/week), we stopped the trial short of our target 50 patients. $84 \%(95 \% \mathrm{Cl} 0.73,0.96)$ of patients received study treatment within 2 hours of the first furosemide dose, $69 \%$ $(95 \% \mathrm{Cl} 0.54,0.80)$ received the complete study regimen, and $20 \%$ received openlabel albumin $(95 \% \mathrm{Cl} 0.1 \mathrm{I}, 0.34)$.

The albumin group had a greater increase in serum albumin (MD $5.94 \mathrm{~g} / \mathrm{L}, 95 \% \mathrm{Cl}$ $[\mathrm{I} .44,6.89], \mathrm{p}=0.0047$ ) and colloid osmotic pressure (MD $3.13 \mathrm{mmHg}, 95 \% \mathrm{Cl}$ $[\mathrm{I} .96,4.3 \mathrm{I}], \mathrm{P}<0.00 \mathrm{I})$. The groups had similar changes in urine output (MD -422 $\mathrm{mL}, 95 \% \mathrm{Cl}[-1062,218], \mathrm{p}=0.20$ ) and fluid balance (MD I47 mL 95\% Cl[-767, $106 \mid], p=0.75)$.

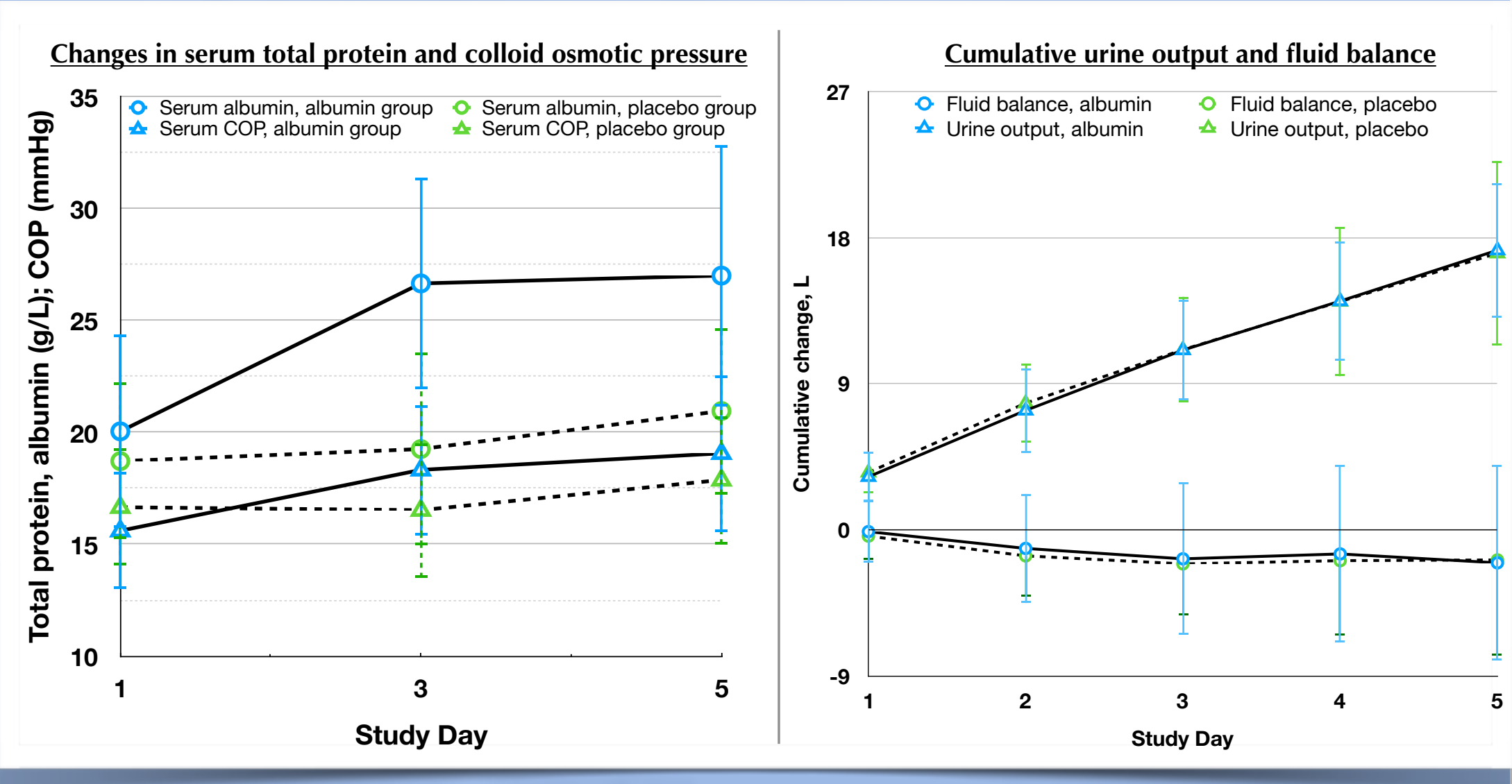

\section{Conclusions}

The current study design was infeasible with respect to rate of recruitment, timely and complete administration of study treatment, and avoidance of openlabel $25 \%$ albumin. With modifications to address these challenges, this pilot study can inform the protocol for a successful definitive trial.
Trial Registration:

Clinicaltrials.gov NCT02055872; ISRCTN 7019188I

Funding source:

Hamilton Health Sciences New Investigator Fund 